\title{
Nerve growth factor signaling following unilateral pelvic ganglionectomy in the rat ventral prostate is age dependent
}

\author{
Carol A Podlasek $^{1}$, Rudrani Ghosh ${ }^{1}$, Omer Onur Cakir ${ }^{1}$, Christopher Bond ${ }^{1}$, Kevin E McKenna ${ }^{2}$ \\ and Kevin T McVary ${ }^{3}$
}

Benign prostatic hyperplasia (BPH) is a serious health concern and is an underlying cause of lower urinary tract symptoms (LUTS) in many men. In affected men, LUTS/BPH is believed to result from benign proliferation of the prostate resulting in bladder outlet obstruction. Postnatal growth of the prostate is controlled via growth factor and endocrine mechanisms. However, little attention had been given to the function of the autonomic nervous system in prostate growth and differentiation. Nerve growth factor (NGF) is a prostatic mitogen that has a trophic role in autonomic sensory end organ interaction. In this study, we examine how the autonomic nervous system influences prostate growth as a function of age by quantifying NGF in the rat ventral prostate (VP) after pelvic ganglionectomy. Unilateral pelvic ganglionectomy was performed on postnatal days 30 (P30), 60 and 120 Sprague-Dawley rats in comparison to sham controls $(n=39)$. Semiquantitative RT-PCR, Western blotting and immunohistochemical analysis for NGF were performed on denervated, intact (contralateral side) and sham control VP 7 days after surgery. Ngf RNA expression was significantly increased in the denervated and intact hyperplastic VP. Western blotting showed age-dependent increases in NGF protein at P60 in the contralateral intact VP. NGF was localized in the nerves, basal cells and columnar epithelium of the prostatic ducts. Denervation causes age-dependent increases in NGF in the VP, which is a potential mechanism by which the autonomic nervous system may regulate prostate growth and lead to BPH/LUTS.

Asian Journal of Andrology (2013) 15, 764-769; doi:10.1038/aja.2013.59; published online 22 July 2013

Keywords: denervation; nerve growth factor; prostate growth; ventral prostate

\section{INTRODUCTION}

Benign prostatic hyperplasia (BPH) is a serious health concern worldwide and is an underlying cause of many lower urinary tract symptoms (LUTS). LUTS is common in aging men and a population based survey performed in five countries reports that $\sim 64 \%$ of adults experience at least one LUTS symptom. ${ }^{1}$ LUTS has a profound impact on social functioning and quality of life in affected men $^{2,3}$ with similar effects reported as after a heart attack or stroke. ${ }^{4} \mathrm{BPH}$ can induce acute urinary retention, incontinence, recurrent urinary tract infection or obstructive uropathy. LUTS secondary to BPH generally consists of three components: benign proliferation of the stroma and epithelium, bladder outlet obstruction and symptoms resulting from obstruction. ${ }^{5}$ $\mathrm{BPH}$ has been considered to be one of the most important risk factors for LUTS; ${ }^{6}$ however, it is becoming increasingly recognized that the origin of LUTS is multifactorial resulting from more than prostate enlargement. ${ }^{7,8}$ We propose that the autonomic nervous system plays an important role in the development of LUTS.

The mammalian prostate is densely innervated by hypogastric and pelvic nerves that play an important role in regulating the growth and function of the gland. The autonomic nervous system has an underappreciated, yet defined role in regulating prostate growth under normal and abnormal conditions. Previous work from our lab supports a significant role of the autonomic nervous system in promoting both prostatic hyperplasia and atrophy by selective neural manipulation. ${ }^{9,10}$ Clinical findings, which complement our previous animal studies, demonstrate a quantitative relationship between the level of autonomic sympathetic activity and LUTS/BPH in symptomatic men. ${ }^{11}$ Thus, the enhanced excitation of the sympathetic nervous system may be a common mechanism underlying BPH and LUTS.

How BPH develops and its relationship to normal growth remains unclear. However, growth and development of the prostate require complex interaction of endocrine pathways, growth factors and stromal-epithelial interactions. ${ }^{12}$ In the search for contributing factors which regulate prostate growth, we focused on factors that respond to neuronal signals and innervation, that function through or in sensory fibers, have a central function in organ-neural development and are localized in the autonomic nervous system. We identified nerve growth factor (NGF) as a potential regulator of prostate growth because of its role as a prostatic mitogen and because of its trophic function in autonomic sensory end organ interaction. ${ }^{13}$ NGF is a neurotrophin, one of a small group of dimeric proteins that critically affect the development of the vertebrate nervous system. The end product of NGF biosynthesis is $7 \mathrm{~S}$ NGF, which contains the $\beta$-NGF

${ }^{1}$ Department of Urology, Northwestern University, Feinberg School of Medicine, Chicago, IL 60611, USA; ${ }^{2}$ Department of Physiology, Northwestern University, Feinberg School of Medicine, Chicago, IL 60611, USA and ${ }^{3}$ Division of Urology, Southern Illinois University School of Medicine, Springfield, IL 62794, USA Correspondence: Dr C Podlasek (cap325@northwestern.edu)

Received: 21 December 2012; Revised: 12 March 2013; Accepted: 20 April 2013; Published online: 22 July 2013 
dimer, the trypsinlike $\gamma$-subunit, and the acidic $\alpha$-subunit. The mature form of NGF, $\beta$-NGF (examined in this study), occurs as a homodimer of two identical subunits, with each subunit $\sim 13 \mathrm{kD}$ in size and the subunits are linked by disulfide bonds.

The prostate is the second most abundant source of NGF after the central nervous system. ${ }^{14}$ Its receptors are implicated in the control of prostate cell proliferation and apoptosis and it can either support or suppress growth. ${ }^{15}$ The coexpression of both NGF receptors, p75 (NGFR) and tropomyosin-related kinase A (TrkA), is crucial for the antiproliferative effect of NGF. P75 (NGFR) is progressively lost during prostate tumorigenesis. Its disappearance represents a malignancy marker of prostate adenocarcinoma and dysregulation of NGF signal transduction was identified in a number of human tumors. ${ }^{15}$ NGF might also contribute to benign proliferation, such as occurs in chronic prostate and chronic pelvic pain syndrome, since NGF abundance in prostate secretions changed in proportion to pain severity. Thus, NGF may be useful as a novel biomarker for chronic prostate and chronic pelvic pain syndrome. In addition to NGF's effects on proliferation, NGF is critical to peripheral nervous system development. It has been proposed that increased $N g f$ in the urinary bladder may contribute to irritative (storage) symptoms in patients with LUTS. NGF regulates nerve growth and survival and induces hyperalgesia and proliferation of afferent sensory neurons. Increased NGF leads to irritative bladder symptoms by overly activating afferent neurons in the sensory system and a recent study reported that NGF increased in the bladder of animals with hyperactive voiding. Bladder outlet obstruction stimulates an increase in bladder NGF, which triggers morphologic and physiological alterations in afferent and efferent neurons. These results suggest that increased $\mathrm{Ng}$ is associated with clinical BPH symptoms. ${ }^{16}$

In order to determine if NGF also plays a role in abnormal prostate growth, we examined the effects of innervation on NGF abundance and localization in sham, denervated and intact (contralateral) ventral prostate (VP) after pelvic ganglionectomy. We propose that denervation on one side of the VP increases prostate innervation and autonomic hyperactivity in the contralateral non-perturbed VP, which may result in increased growth. This study will offer insight as to how age related growth plasticity affects basic pathophysiological processes, which regulate prostate morphology and thus provide a mechanism as to how men with autonomic hyperactivity may develop LUTS/BPH.

\section{MATERIALS AND METHODS}

\section{Animals}

Thirty-nine Sprague-Dawley rats postnatal day 30 (P30, $n=12$ ), P60 $(n=12)$ and P120 $(n=15)$ were obtained from Harlan Laboratories (Cumberland, IN, USA). The weight range of the rats is $76-100 \mathrm{~g}$ for P30, 251-275 g for P60 and 400 g for P120.

\section{Ethics statement}

All animals were cared for in accordance with institutional IACUC approval and the National Research Council publication Guide for Care and Use of Laboratory Animals.

\section{Denervation surgery}

A midline lower abdominal incision was made in Sprague-Dawley rats to expose the pelvic ganglia (PG). For the denervated group $(n=18)$, glass nerve hooks and microscissors were used to perform a unilateral pelvic ganglionectomy, removing the left PG (Figure 1). Sham control surgeries were performed in age-matched controls by exposing the PG without excision $(n=18)$. To eliminate potential stress-related

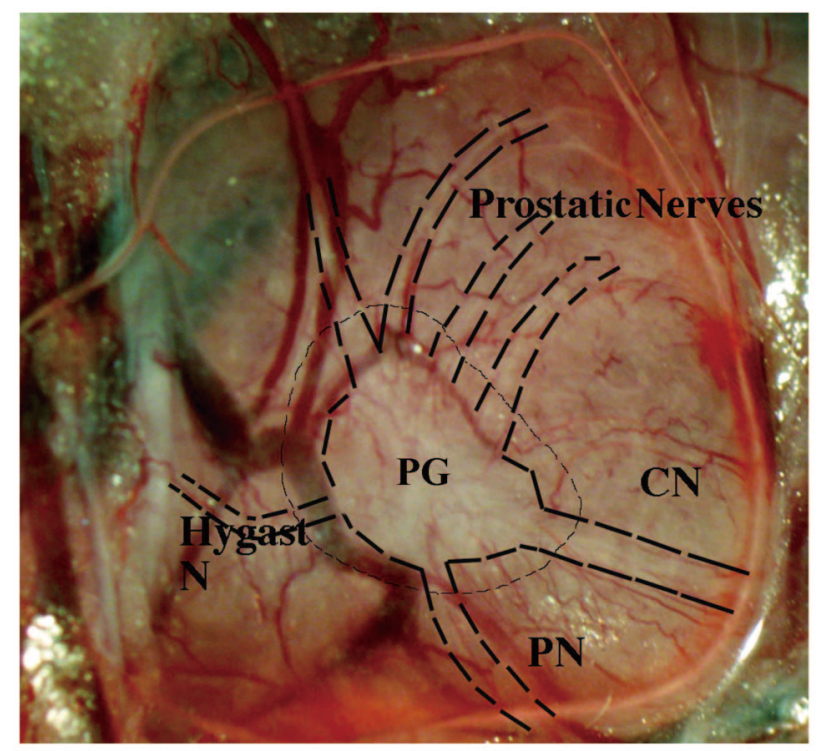

Figure 1 Photo depicting the pelvic plexus and nerves that innervate the prostate and penis. A dotted line depicts the tissue removed during pelvic ganglionectomy surgery. $\times 100$ magnification. $\mathrm{CN}$, cavernous nerve; Hygast $\mathrm{N}$, hypogastric nerve; PG, pelvic ganglia; PN, pelvic nerve.

fluctuations of serum testosterone, bilateral epididymo-orchiectomy was performed and a 3 - $\mathrm{cm}$ piece of medical-grade silastic tubing (Dow Corning Corp, Midland, MI, USA) filled with crystalline testosterone (Sigma, St Louis, MO, USA) was placed subcutaneously at the time of surgery. This method ensures uniform serum testosterone levels for all groups. ${ }^{17}$ The concentration of testosterone present in vivo in rats with age $^{18}$ and the number of centimeters of testosterone stick required to bring testosterone levels back to physiological levels after castration has previously been determined. ${ }^{19}$ Denervated $(n=18)$, intact (contralateral side, $n=18)$ and sham control $(n=18)$ operated rats were sacrificed 7 days after surgery and the VP were excised and either frozen in liquid nitrogen or fixed in $4 \%$ paraformaldehyde. Seven days was chosen for study since in vitro studies demonstrated an impact on NGF exposure on neurite and microtubule length and protein abundance at 7 days. ${ }^{20}$

\section{RNA isolation and reverse transcriptase-polymerase chain reaction} (RT-PCR)

Total RNA was isolated from P30, P60 and P120 denervated $(n=12)$, intact $(n=12)$ and sham control $(n=12)$. VP using the Trizol method (Life Technologies, Grand Island, NY, USA) and the RNA was deoxyribonuclease-treated to eliminate any genomic DNA contamination. Semi-quantitative RT-PCR was performed to quantify Ngf on 150 200 ng total RNA using the Gene Amp RNA PCR core kit (PerkinElmer, Branchburn, NJ, USA) as described previously using noncompetitive methodology and Gapdh as an endogenous internal standard. ${ }^{21,22}$ Primers were synthesized at the Northwestern Biotechnical Facility ( $N g f$ sense: $5^{\prime}$-AAG TTA TCC CAG CCA AAC TA-3', Ngf anti-sense: 5'-ATG TCA GTG TTG GGA GTA GG-3', Gapdh sense: 5'-GTC GGT GTC AAC GGA TTT G-3', Gapdh antisense: 5'-ACA AAC ATG GGG GCA TCA G-3'). Products were restriction-digested to confirm that the bands represented the sequences of interest. Quantification was performed 6 times on individual tissue specimens and the product ratios were averaged and reported as the mean \pm standard error of the mean. 


\section{Western blotting analysis}

P30, P60 and P120 denervated $(n=15)$, intact $(n=15)$ and sham control $(n=15)$ VPs were homogenized individually in PBS with protease inhibitors (PMSF, EDTA, Leupetin), diluted in $2 \times$ sample buffer $\left(10 \mathrm{mmol} \mathrm{l}^{-1}\right.$ Tris, 4\% SDS, $200 \mathrm{mmol} \mathrm{l}^{-1}$ dithiothreitol, $20 \%$ glycerol, $0.02 \%$ bromophenol blue) and heated at $95{ }^{\circ} \mathrm{C}$ for $5 \mathrm{~min}$. Proteins were quantified by the Lowry method and $100 \mu \mathrm{g}$ protein was run per lane on a $10 \%$ polyacrylamide gel. Proteins were transferred to a PVDF membrane using a Hoefer Semi-phor Semi-dry electroblotter, for $2 \mathrm{~h}$. After blocking overnight with 5\% powdered milk in PBS at $37{ }^{\circ} \mathrm{C}$, membranes were incubated with rabbit polyclonal NGF (Millipore, Chemicon, CA, USA) or mouse $\beta$-ACTIN (Sigma) antibodies for $18 \mathrm{~h}$ at $4{ }^{\circ} \mathrm{C}$. Membranes were treated with horseradish peroxidase conjugated chicken anti-mouse (1/8000) or chicken anti-rabbit (1/5000; Santa Cruz, Santa Cruz, CA, USA) secondary for $1 \mathrm{~h}$ at $37^{\circ} \mathrm{C}$. Protein bands were visualized using 3,3',5,5'-Tetramethylbenzidene perioxidase substrate (KPL, Garthersburg, MD, USA) and were quantified by densitometry using Kodak 1D software (Rochester, NY, USA). The ratio of NGF/ $\beta$-ACTIN was determined for individual tissues in triplicate and tissues were averaged and reported \pm standard error of the mean.

\section{Immunohistochemical analysis (IHC)}

IHC was performed on frozen sections $(14 \mu)$ of normal VP $(n=3)$. Optimal cutting temperature compound (OCT, Tissue-Tek, Torrance, CA, USA) was removed by washing with $1 \times$ phosphate-buffered saline (PBS; Life Technologies). Sections were blocked using 3\% dry milk in PBS for $1 \mathrm{~h}$ at room temperature. Sections were incubated with rabbit anti-NGF- $\beta$ polyclonal antibody (1/50; Millipore) overnight at $4{ }^{\circ} \mathrm{C}$. Negative controls were performed in the absence of primary antibody and using rabbit serum. After washing, sections were treated with Alexa Fluor 488 chicken anti-rabbit (1/200; Molecular Probes, Carlsbad, CA, USA). Sections were dehydrated and mounted using DPX Mounting Media (Hatfield, PA, USA). Dual staining was performed with NGF (as stated above) and neuron-specific enolase (1/100; Millipore) using Alexa Fluor 594 donkey anti-mouse (1/250; Life Technologies) secondary antibody.

\section{Statistical analysis}

Statistical analysis was performed using ANOVA and Scheffe's post hoc test and results were reported \pm standard error of the mean. Differences were considered significant when $P \leqslant 0.05$.

\section{RESULTS}

Quantitation of Ventral Prostate Ngf RNA expression by RT-PCR Ngf RNA expression was quantified by RT-PCR in P30, P60 and P120 denervated $(n=12)$, intact $(n=12)$ and sham control $(n=12)$ VP, 7 days after unilateral pelvic ganglionectomy (Figure 2). When pelvic ganglionectomy was performed on P30 rats, there was a significant $50 \%$ increase in $\mathrm{Ng}$ expression in the denervated VP in comparison to sham controls $(P=0.0018)$, a $39 \%$ increase in the intact VP in comparison to sham controls $(0.0018)$ and there was no significant difference between the denervated and intact VP $(P>0.05)$. When pelvic ganglionectomy was performed on $\mathrm{P} 60$ rats, there was a significant $42 \%$ increase in $\mathrm{Ngf}$ expression in the denervated VP in comparison to sham controls ( $P=0.0006$, Figure 2$)$, a $27 \%$ increase in the intact VP in comparison to sham controls $(P=0.0006$, Figure 2$)$ and a $20 \%$ increase in the denervated VP in comparison to the intact VP $(P=0.0006)$. When pelvic ganglionectomy was performed on $\mathrm{P} 120$ rats, there was a significant $70 \%$ increase in $N g f$ expression in the denervated VP in comparison to sham controls $(P=0.0116$, Figure 2), no significant difference between the intact VP in comparison to sham controls $(P>0.05$, Figure 2$)$ and a significant $74 \%$ increase in the denervated versus intact VP $(P=0.0116$, Figure 2$)$.

\section{Quantification of NGF protein abundance by Western blotting analysis}

NGF protein was quantified in P30, P60 and P120 denervated $(n=15)$, intact $(n=15)$, and sham control $(n=15)$ VP 7 days after unilateral pelvic ganglionectomy (Figure 3 ). When pelvic ganglionectomy was performed on P30 rats, there was no difference in NGF protein between the three groups (Figure 3, $P=0.9113$ ). However, pelvic ganglionectomy in P60 rats caused a significant $15 \%$ increase in NGF in the intact VP in comparison to the sham control VP $(P=0.0368$, Figure 3$)$. There was no difference between the denervated and sham and the intact and denervated VP ( $P>0.05$, Figure 3). When pelvic ganglionectomy was performed on P120 rats, ANOVA showed a significant difference in the groups $(P=0.0402)$; however, Scheffe's posthoc test, which is more conservative than the ANOVA, did not identify differences between the sham, denervated and intact VPs (Figure 3). For discussion of non-significant posthoc tests in the presence of a significant F-test, see Cardinal and Aitken (2006). ${ }^{23}$ The lack of NGF response in the P120 intact group suggests a loss of neural plasticity with age.

\section{IHC analysis of NGF protein localization in normal, adult VP}

IHC analysis was performed to localize NGF protein in normal adult VP $(n=3)$. NGF protein was localized in nerves present in the stroma
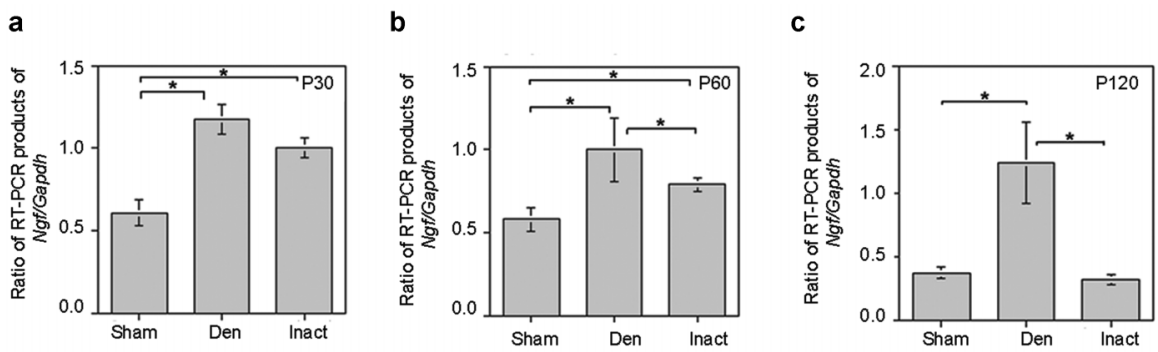

Figure 2 Quantification of Ngf/Gapdh RNA expression in denervated, intact and sham control VP. (a) In P30 rats, Ngf increased $50 \%$ in the denervated VP in comparison to sham controls ( $P=0.0018)$, increased $39 \%$ in the intact VP in comparison to sham controls $(P=0.0018)$, and there was no difference between the denervated and intact VP ( $P>0.05)$. (b) In P60 rats, Ngfincreased $42 \%$ in the denervated VP in comparison to sham controls ( $p=0.0006)$, increased $27 \%$ in the intact VP in comparison to sham controls ( $P=0.0006)$, and increased $20 \%$ in the denervated VP in comparison to the intact VP $(P=0.0006)$. (c) In $\mathrm{P} 120$ rats, Ngfincreased $70 \%$ in the denervated VP in comparison to sham controls $(P=0.0116)$, increased $74 \%$ in the denervated VP in comparison to the intact VP $(P=0.0116)$ and there was no difference between the intact and sham control VPs $(P>0.05)$. Asterisks indicate significant differences. Den, denervated; VP, ventral prostate. 
a

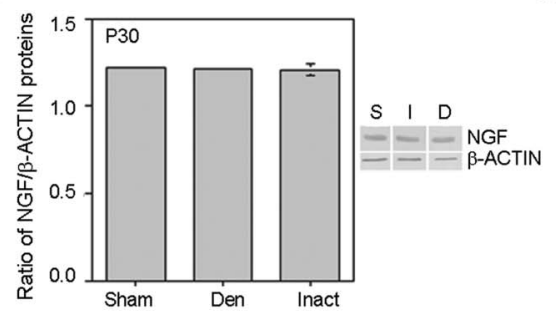

b

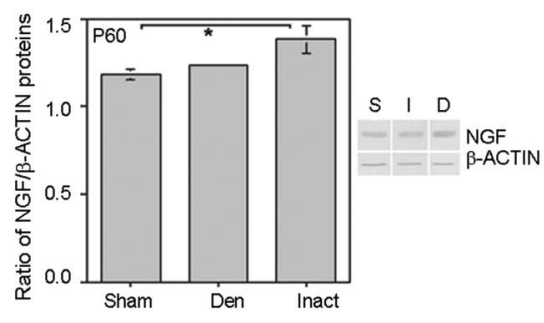

c

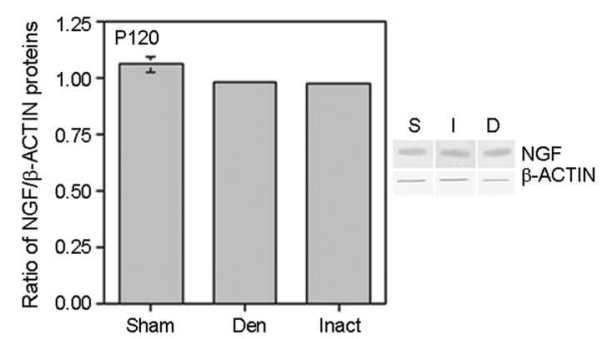

Figure 3 Quantification of NGF/ $\beta$-ACTIN proteins in denervated, intact and sham control VP. (a) In P30 rats, there was no difference in NGF protein between the three groups ( $P=0.9113$ ). (b) In P60 rats, NGF increased $15 \%$ in the intact VP in comparison to sham control VP $(P=0.0368)$. There was no difference between the denervated and sham and the intact and denervated VP $(P>0.05)$. (c) In P120 rats, although ANOVA resulted in $P=0.0402$, Scheffe's posthoc test did not find difference between the sham, denervated and intact VP. Asterisks indicate significant differences. Where error bars are not present the standard error of the mean is too small for the error bars to graph. Den, denervated; D, denervated; I, Intact; NGF, nerve growth factor; S, sham; VP, ventral prostate.

and in the ductal epithelium (Figure $4 a$ and $4 b$ ). Staining was particularly abundant in the basement membrane surrounding the prostatic ducts (Figure 4a). Dual staining was performed with neuron specific enolase and NGF which showed colocalization of NGF and neuron specific enolase in VP nerves (Figure 4c).

\section{DISCUSSION}

Stromal-epithelial interactions play a central role in normal and abnormal growth of the prostate. The prostate consists of ducts lined with epithelium and stromal cells (including fibroblasts and smooth muscle cells), which lie in between the ducts. ${ }^{24}$ Prostate epithelium includes basal cells, columnar secretory cells and neuroendocrine cells, which are androgen-dependent. ${ }^{25,26}$ Our findings localize $\beta$-NGF to
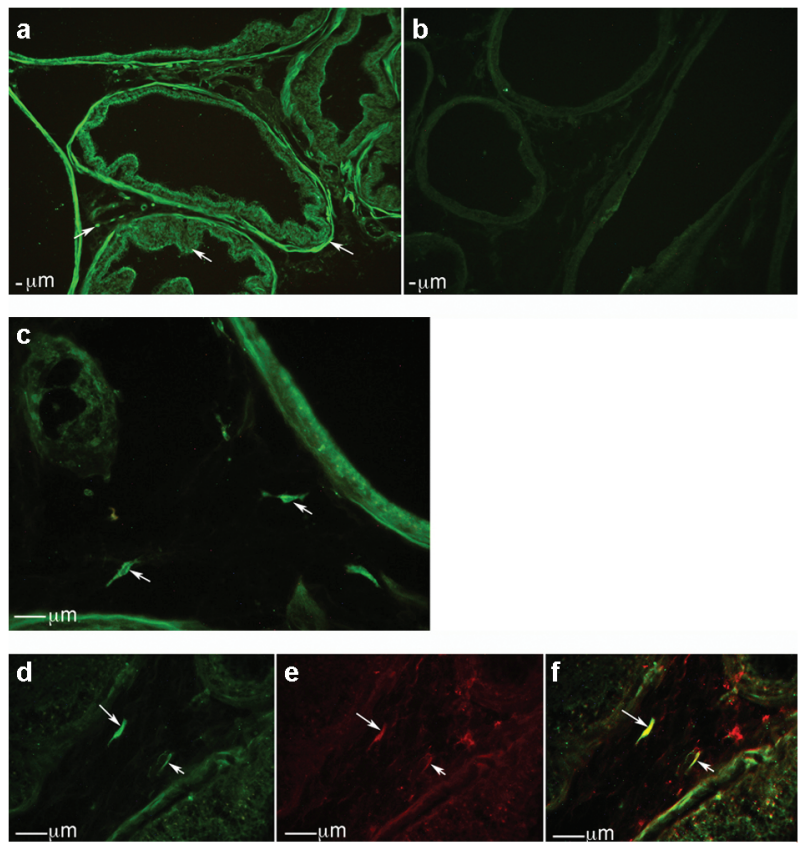

Figure $4 \mathrm{IHC}$ analysis of NGF protein in normal, adult Sprague Dawley VP (a) in comparison to negative controls (b). (a) NGF is localized in nerves, ductal epithelium and basal cells surrounding the ducts. (c) Magnified view of NGF protein in nerves present in the stroma. NGF (green, d) colocalizes with neuron specific enolase (red, $\mathbf{e}$ ) in neurons of the VP. Colocalization is shown in yellow (f). Arrows indicate staining for NGF and/or neuron specific enolase respectively. Scale bars $=10 \mu \mathrm{m}$. NGF, nerve growth factor; VP, ventral prostate. nerves of the prostate, and basal cells and columnar epithelium of the prostatic ducts. Previous morphological studies suggest that the stroma plays a critical role in affecting the growth and maturation of the prostatic epithelium ${ }^{27}$ and that NGF is a secreted protein with its receptors located in the epithelium. ${ }^{28}$ We propose that it is the nerves of the prostate that are the origin of NGF which interacts with its receptors in the epithelium, and thereby constitutes a mechanism whereby the autonomic nervous system impacts prostate morphogenesis (manipulation of NGF abundance).

Our RT-PCR results show that Ngfexpression is elevated in both the denervated and the contralateral neurally intact VP in response to denervation at P30 and P60. The increase in both intact and denervated $N g f$ gene expression reflects the bilateral crossover of prostate innervation as previously demonstrated. ${ }^{10}$ In addition at P60 the denervated group is further increased $20 \%$ over the intact group. At $\mathrm{P} 120$, the denervated VP was significantly increased relative to the sham and intact group, while the intact VP showed no significant change in $\mathrm{Ng}$ in comparison to the sham. These results were confirmed by Western blotting analysis in which NGF protein was increased in the intact VP at P60 but not at P120. The lack of response of the intact VP in the adult suggests an age-related loss of neural plasticity. This idea is supported by observations during development that the presence of NGF in defined target areas attracts axonal growth and synapses and secures the eventual survival of NGF-dependent neurons. However, in the mature nervous system, NGF secures phenotypic maintenance of NGF-dependent neurons rather than their survival. A gradual increase in $N g f$ and $T r k A$ expression also occurs with age. ${ }^{29,30}$ These findings are consistent with a contribution of NGF to the change in autonomic tone observed in elderly patients. ${ }^{30}$

At 60 days, NGF protein was significantly increased in the intact VP versus the sham despite the fact that $N g f$ RNA expression was highest in the denervated VP. This increase in intact NGF protein suggests that with nerve perturbation, the adolescent, neurally intact prostate has both the appropriate neural signal and an undiminished capacity for epithelial response. This may reflect an effect from the crossover of innervation from the impaired side as well as the intactness of the ipsilateral nerves. This increased NGF has an internal consistency in so far as it is this intact group that demonstrated a hyperplastic response following several types of nerve manipulation. ${ }^{9}$ This undiminished capacity for intact side stromal response may explain the hyperplastic VP response noted in our denervation injury model as well as that seen in the spontaneously hypertensive rat. ${ }^{9,31}$ Although not necessarily a causal relationship, the induction of intact side prostatic hyperplasia with nerve manipulation concurrently with 
increased NGF is interesting from the neural growth regulation perspective. Whether there is a causal relationship between the hyperplastic response and increased VP NGF protein abundance will require additional studies. Induction of a trophic factor in the in vivo rat prostate by neural manipulation may be an important pathogenic mechanism in disease states of adulthood. Given that target organ NGF levels would likely increase with nerve perturbation supports our line of investigation. This inducible NGF response is hypothesized to occur in an attempt to re-establish appropriate innervation and requires a responsive and plastic neural control mechanism as well as target tissue that can produce sufficient signal to promote a neural response.

The lack of NGF response in the P120 intact group is different than that noted with the younger groups and may reflect a loss of neuralepithelial plasticity in the older age group. It appears that the intact side has a limited time frame in which neural influences produce increases in the prostatic mitogen. Epithelial-neural interactions may have a critical time period in which they are active and available to influence prostate growth. It is interesting that this time period approximates the adolescent time frame, a physiologic period in which hyperplastic response is most critical. Determining whether the hyperplastic response of the VP is mediated by NGF, in addition to other growth factors, requires further study. This age-related effect might be explained by the differences in neural resilience, interaction between prostatic epithelium and the autonomic nervous system or more fundamental changes in the prostatic epithelium response to neural perturbation. We favor the idea that age affects the plasticity of the nerves and the signaling pathways by which they control prostate growth. It is also possible that a 7-day post-surgical waiting period is not sufficient to show changes in protein abundance in the P30 and P120 VP.

In the context of our recent findings, we hypothesize that perturbation of the neuroregulatory mechanism leads to an increase in prostate innervation and autonomic hyperactivity in the nearby non-perturbed intact (contralateral) prostate tissue. This results in increased growth and a gland-wide increase in NGF and possibly other mitogens. These neuronal regulatory changes may be key factors resulting in prostatic hyperplasia. Complementing our basic science study is clinical work in which a quantitative relationship between the level of autonomic sympathetic activation and LUTS/BPH measures in symptomatic men was demonstrated. ${ }^{11}$ Previous epidemiological studies in men with $\mathrm{BPH}$ suggest there may be a relationship between sympathetic overactivity and development of LUTS. Meigs et al. ${ }^{32}$ noted that men with symptomatic BPH were more likely to also have characteristics associated with increased sympathetic activation, specifically, heart disease, betablocker use and a sedentary lifestyle. Hammarsten et al. ${ }^{33}$ also found a relationship between faster-developing $\mathrm{BPH}$ and diseases associated with sympathetic over activity. Clinical studies investigating the role of neural influences on LUTS/BPH are underway. ${ }^{34}$

The presence of an age limited neural-epithelial response following neural perturbation is an interesting finding in our model. Several investigators have demonstrated reduced age-related plasticity of neurotrophin receptor expression in selected sympathetic neurons of the rat. ${ }^{35}$ Age-related reduction in neuronal sprouting is speculated to reflect increasing commitment to information storage at the expense of neuronal plasticity. ${ }^{35}$ Whether a similar rationale exists in an increasingly metabolically active 120 -day VP is an interesting conjecture.

\section{CONCLUSION}

These results show that perturbation of prostatic innervation by selective pelvic ganglionectomy induced a significant increase in $\mathrm{Ngf}$ in both the neurally intact hyperplastic tissue as well as in the denervated atrophied ventral prostate. The changes in NGF protein abundance varied according to nerve intactness and age. Increased NGF, a growth factor with known mitogenic potential, in non-perturbed prostate, is a potential mechanism by which the autonomic nervous system may influence prostate growth and the induction of hyperplasia. Studies focusing on altering neuronal activity in order to treat BPH/LUTS are underway.

\section{AUTHOR CONTRIBUTIONS}

KTM, CAP, KEM and RG designed the research. CAP, KTM and RG wrote the paper. RG, CAP, CB and OOC carried out the studies and performed statistical analysis. All authors read and approved the final manuscript.

\section{COMPETING FINANCIAL INTERESTS}

All authors declare that there are no competing financial interests.

\section{ACKNOWLEDGMENTS}

The authors would like to thank John Cashy for statistical analysis. This study was supported by National Institutes of Health/National Institute of Diabetes and Digestive and Kidney Diseases (No. DK079184).

1 Irwin DE, Milsom I, Hunskaar S, Reilly K, Kopp Z et al. Population-based survey of urinary incontinence, overactive bladder, and other lower urinary tract symptoms in five countries: results of the EPIC study. Eur Urol 2006; 50: 1306-14.

2 Grayhack JT, McVary KT, Kozlowski JM. Benign prostatic hyperplasia. In: Gillenwater JJ, Grayhack JT, Howards SS, Mitchell ME, editors. Adult and Pediatric Urology. Vol. 2, 4th ed. Philadelphia, PA: Lippincott Williams and Wilkins Publishers; 2001. p1401-70.

3 Girman CJ, Jacobsen SJ, Tsukamoto T, Richard F, Garraway WM et al. Health-related quality of life associated with lower urinary tract symptoms in four countries. Urology 1998; 51: 428-36

4 Robertson $\mathrm{C}$, Link CL, Onel E, Mazzetta C, Keech M et al. The impact of lower urinary tract symptoms and comorbidities on quality of life: the BACH and UREPIK studies. BJU Int 2007; 99: 347-54.

5 Bang WJ, Kim HW, Lee JY, Lee DH, Hah YS et al. Prostatic urethral angulation associated with urinary flow rate and urinary symptom scores in men with lower urinary tract symptoms. Urology 2012; 80: 1333-7.

6 Girman CJ, Jacobson SJ, Guess HA, Oesterling JE, Chute CG et al. Natural history of prostatism: relationship among symptoms, prostate volume and peak urinary flow rate. J Urol 1995; 153: 1510-5.

7 Wade B. Eitology, epidemiology, and natural history. Urol Clin North Am 2009; 36: 403-15.

8 Fowke JH, Phillips S, Koyama T, Byerly S, Concepcion R et al. Association between physical activity, lower urinary tract symptoms (LUTS) and prostate volume. BJU Int 2013; 111: 122-8.

9 McVary KT, Razzaq A, Lee C, Venegas MF, Rademaker A et al. Growth of the rat prostate gland is facilitated by the autonomic nervous system. Biol Reprod 1994; 51: 99-107.

10 McVary KT, McKenna KE, Lee C. Prostate innervation. Prostate Supp/ 1998; 8: 2-13.

11 McVary KT, Rademaker A, Lloyd GL, Gann P. Autonomic nervous system overactivity in men with lower urinary tract symptoms secondary to benign prostatic hyperplasia. J Urol 2005; 174(4 Pt 1): 1327-433.

12 Podlasek CA, Barnett DH, Clemens JQ, Bak PM, Bushman W. Prostate development requires Sonic hedgehog expressed by the urogenital sinus epithelium. Dev Biol 1999; 209: 28-39.

13 Djakiew D. Role of nerve growth factor-like protein in the paracrine regulation of prostate growth. J Androl 1992; 13: 476-87.

14 Papatsoris AG, Liolitsa D, Deliveliotis C. Manipulation of the nerve growth factor network in prostate cancer. Expert Opion Investig Drugs 2007; 16: 303-9.

15 Arrighi N, Bodei S, Zani D, Simeone C, Cunico SC et al. Nerve growth factor signaling in prostate health and disease. Growth Factors 2010; 28: 191-201.

16 Kim JC, Park EY, Hong SH, Seo SI, Park YH et al. Changes of urinary nerve growth factor and prostaglandins in male patients with overactive bladder symptoms. Int $J$ Urol 2005; 12: 875-80.

17 Wang JM, McKenna KE, McVary KT, Lee C. Requirement of innervation for maintenance of structural and functional integrity in the rat prostate. Biol Reprod 1991; 44: 1171-6.

18 Korenbrot CC, Huhtaniemi IT, Weiner RI. Preputial separation as an external sign of pubertal development in the male rat. Biol Reprod 1977; 17: 298-303. 
19 Dalton DP, Lee C, Huprikar S, Chmiel JS, Grayhack JT. Non-androgenic role of testis in enhancing ventral prostate growth in rats. Prostate 1990; 16: 225-33.

20 Drubin DG, Feinstein SC, Shooter EM, Kirschner MW. Nerve growth fator-induced neurite outgrowth in PC12 cells involves the coordinate induction of microtubule assembly and assembly-promoting factors. J Cell Biol 1985; 101: 1799-807.

21 Seibert P. Quantitative RT-PCR, Clontech Methods and Applications, Book 3. Palo Alto, CA: Clontech Laboratories; 1993.

22 Podlasek CA, Duboule D, Bushman W. Male accessory sex organ morphogenesis is altered by loss of function of Hoxd-13. Dev Dyn 1997; 208: 454-65.

23 Cardinal RN, Aitken MR. ANOVA for the Behavioral Sciences Researcher. Mahwah, NJ: Lawrence Erlbaum Associates; 2006. p91.

24 Coffey, DS. The molecular biology of the prostate. In: Lepor H, Lawson RK, editors. Prostatic Diseases. Philadelphia, PA: W.B. Saunders; 2000. p28-56.

25 Habermann H, Chang WY, Birch L, Mehta P, Prins GS. Developmental exposure to estrogens alters epithelial cell adhesion and gap junction proteins in the adult rat prostate. Endocrinology 2001; 142: 359-69.

26 Risbridger G, Wang H, Young P, Kurita T, Wong YZ et al. Evidence that epithelial and mesenchymal estrogen receptor- $\alpha$ mediates effects of estrogen on prostatic epithelium. Dev Biol 2001; 229: 432-42.

27 Lopaczynski W, Hruszkewycz AM, Lieberman R. Preprostatectomy: a clinical model to study stromal-epithelial interactions. Urology 2001; 57(Suppl 4A): 194-9.
28 Graham CW, Lynch JH. Djakiew D. Distribution of nerve growth factor-like protein and nerve growth factor receptor in human benign prostatic hyperplasia and prostatic adenocarcinoma. J Urol 1992; 147: 1444-7.

29 Chen ZP, Zhou HL, Cao LS, Gao R. Significance of nerve growth factor expression in the prostate tissue of spontaneously hypertensive rats. Zhonghua Nan Ke Xue 2011 , 17: 781-4.

30 Sayfili E, Kluttig R, Rana OR, Saygili E, Gemein C et al. Age-related regiona differences in cardiac nerve growth factor expression. Age (Dordr) 2012; 34: 659-67.

31 Persson K, Pandita RK, Spitsbergen JM, Steers WD, Tuttle JB et al. Spinal and peripheral mechanisms contributing to hyperactive voiding in spontaneously hypertensive rats. Am J Physiol 1998; 275(4 Pt 2): R1366-73.

32 Meigs JB, Mohr B, Barry MJ, Collins MM, McKinlay JB. Risk factors for clinical benign prostatic hyperplasia in a community-based population of healthy aging men. J Clin Epidemiol 2001; 54: 935-44.

33 Hammarsten J, Hogstedt B. Clinical, anthropometric, metabolic and insulin profile of men with fast annual growth rates of benign prostatic hyperplasia. Blood Press 1999; 29-36.

34 Marberger M, Chartier-Kastler E, Egerdie B, Lee KS, Grosse J et al. A randomized double-blind placebo-controlled phase 2 dose-ranging study of Onabotulinumtoxin A in men with benign prostatic hyperplasia. Eur Urol 2013; 63: 496-503.

35 Lim KC, Lim ST, Federoff HJ. Neurotrophin secretory pathways and synaptic plasticity. Neurobiol Aging 2003; 24: 1135-45. 